with Lorenzo oil therapy in ALD is deficient at best. In view of the complexities of fatty acid metabolism, more detailed studies are essential. Especially since "nutripharms are 'naturally' appealing to the general public" (Walker et al, 1999), one should be careful not to generate another "prematurely amplified hope" (Moser, 1993).

Moser, H.W. (1993) Lorenzo oil therapy for adrenoleukodystrophy: a prematurely amplified hope. Annols of Neurology, 34, 121-122.

—, Moser, A. B., Smith, K. D., et al (1992)

Adrenoleukodystrophy: phenotypic variability and implications for therapy. Journal of Inherited Metabolic Disorders, 15, 645-664.

Poulos, A., Gibson, R., Sharp, P., et al (1994) Very long chain fatty acids in X-linked adrenoleukodystrophy brain after treatment with Lorenzo's oil. Annals of Neurology, 36. 741-746.

Walleor, N. P., Fox, H. C. \& Whalley, L. J. (1999) Lipids and schizophrenia. British Journal of Psychiotry, 174. $101-104$.

Zinlcham, W. H., Kiclder, T., Borel, M. S., et al (1993) Lorenzo's oil and thrombocy topenia in patients with adrenoleukodystrophy. New England journal of Medicine. 328, 1126- II27.

I. Maurer, H.-P. Volz Department of Psychiatry, Friedrich-Schiller University Jena, Philosophenweg 3. 07740 Jena, Germany

Authors' reply: Drs Maurer \& Volz offer a helpful overview of ALD, however they take our analogy between schizophrenia and ALD too literally. We aimed to emphasise by example the potential impact of abnormal lipid metabolism on brain function.

The purpose of our editorial was to review the evidence for and against a role of altered lipid handling in schizophrenia. We acknowledge that this is inconclusive but we argue that there is sufficient consistency to make further hypothesis-testing worthwhile. It is true that it would be premature to claim a breakthrough in the treatment of schizophrenia in spite of encouraging case reports (Puri et al, 1998), but it is not premature to postulate.

Purt, B. K., Steiner, R. \& Richardeon, A. J. (1990)

Sustained remission of positive and negative

symptoms of schizophrenia following treatment with eicosapentaenoic acid. Archives of General Psychiotry, 55 $188-189$.

N. P. Walker, H. C. Fox, L. J. Walley

Department of Mental Health, University of Aberdeen, Clinical Research Centre, Royal Cornhill Hospital, Cornhill Road, Aberdeen AB25 2ZH

\section{Pharmacokinetics of clozapine}

Sir: The paper by Kurz et al (1998) attempted to fill a long-neglected gap in our knowledge of the pharmacokinetics of clozapine and has important implications for clinicians who use clozapine levels as a means of optimising therapy. An early paper by Thorup \& Fog (1977) had suggested that intra-patient variability was marked in some patients, but that study had serious methodological flaws. Following Kurz's study we now know that patients on stable doses of clozapine may show considerable variability without clinical deterioration.

What implications does this have for clinicians? Generally, clozapine levels are used in patients who have only a partial response to clozapine, or who relapse after initially responding well. In view of Kurz et al's findings, modifying the dose after checking a single clozapine level is now untenable. Measuring serial levels may be helpful in those patients who can be shown to have little variability, but these appear to be few and far between.

Kurz et al suggest that levels may also be useful in problem patients with levels of variability above $50 \%$, in that these suggest poor compliance. This is a non sequitur. Coefficients of variability above $50 \%$ may represent poor compliance - so may coefficients below $50 \%$. If we are to continue to use clozapine levels in problem patients, two questions need to be answered. First, is clinical deterioration related to fluctuations in clozapine levels in some patients? Second, what causes this variability?

In terms of the first point, Kurz et al have clearly shown that some patients will remain well, even when their levels vary widely. This may not apply to all patients: indeed, exclusion criteria are not specified in this study, but it seems likely that patients who did relapse during the course of the study were excluded for this reason. Checking regular levels in individual patients on clozapine should indicate whether or not they are sensitive to fluctuations.

The second question concerns the cause of the variability in levels. Pharmacokinetic variables are certainly one possibility. I suspect, however, that insufficient consideration has been given to the issue of compliance. Previous studies using various measures of compliance, including pill counts, clinician's estimates and interviews with patients, have assessed compliance in patients on antipsychotic medications at between 24 and 90\% (Falloon et al, 1978; Buchanan, 1992).
The wide range described probably reflects the different methods of assessment used. It is not clear how Kurtz et al attempted to ensure compliance, but direct questioning and clinician's judgement have generally been found to be unreliable (Cramer, 1991). If an in-patient group, whose medication was closely supervised, had much lower mean intra-individual coefficients of variation than those found by Kurz et al, the interpretation of variable plasma levels would be clearer and regular assessments would indeed become a useful guide in the management of problem patients.

Buchanan, A. (1992) A two-year prospective study of treatment compliance in patients with schizophrenia. Psychological Medicine, 22, 787-797.

Cramer, J. A. (1991) Overview of methods to measure and enhance patient compliance. In Potient Compliance in Medical Practise and Clinical Trials (eds J. A. Cramer \& I. A. Spiker). New York: Raven Press.

Falloon, 1., Watt, D. C. \& Shephend, M. (1975) A comparative controlled trial of pimozide and fluphenazine decanoate in the continued treatment of schizophrenia. Psychological Medicine, 8, 59-70.

Kurz, M., Hurnmer, M., Kemmier, G., et of (ISs)e) Long-term pharmacokinetics of clozapine. British fournal of Psychiatry, I73, 341-344.

Thorup, M. \& Fog, R. (1977) Clozapine treatment of schizophrenic patients. Acto Psychiatrica Scandinovico, 55. $123-126$.

G. Swift Department of Psychiatry, Mater Misericordiae Hospital, University College Dublin,

63 Eccles Street, Dublin 7. Republic of Ireland

\section{Medial prefrontal glutamine and dreaming}

Sir: In their review article, Feinberg \& Guazzelli (1999) proposed that malfunctioning corollary discharge and feed-forward systems in the brain could explain many of the symptoms of schizophrenia. Arguments were presented that implicated neuronal circuits involving the basal ganglia, thalamus and prefrontal cortex in this disease. Of particular interest to us were the parallels drawn between dreaming and psychosis.

Our group is using magnetic resonance spectroscopy (MRS) to study the limbic basal ganglia-thalamocortical circuit in subjects with schizophrenia. In a previous study, we found elevated levels of glutamine, a precursor and metabolite of the excitatory neurotransmitter glutamate, in never-treated patients with schizophrenia in the left medial prefrontal cortex, compared with healthy volunteers (Bartha et al, 1997). This is of note because the basal ganglia-thalamocortical 